\title{
Le dépérissement du sapin dans les Vosges: rôle primordial de déficits d'alimentation en eau
}

\author{
G. LÉVY \& M. BECKER * \\ avec la collaboration technique de Y. LEFEVRE \& R. SCHIPFER * \\ INRA, \\ Station de Recherches sur le Sol, la Microbiologie et la Nutrition des Arbres forestiers \\ * Laboratoire de Phyto-écologie forestière \\ Centre de Recherches de Nancy, BP 35, Champenoux, F 54280 Seichamps
}

\begin{abstract}
Résumé
Pour étudier ce dépérissement, une méthode facilitant l'interprétation des différences d'état de santé entre peuplements a été retenue. Huit couples de placettes de six arbres chacune ont été choisis. Les deux placettes de chaque couple sont l'une très saine (s), l'autre très dépérissante (d). Elles sont situées généralement très près l'une de l'autre; le niveau de pollution atmosphérique et les conditions climatiques sont ainsi à peu près identiques dans les deux placettes.

Le sol le plus acide et le moins riche correspond, dans un couple, tantôt à la placette s, tantôt à la placette d.

Des courbes de croissance radiale moyenne annuelle dans les placettes, modifićes pour éliminer l'effet de l'àge, ont été tracées. Elles montrent deux périodes où les accroissements sont faibles, correspondant chacune à 2 ou 3 années sèches : $1959,1962,1964 ; 1974$ et surtout 1976. Mais les accroissements annuels dans les placettes d ont continué à être faibles bien après 1965 . jusqu'au stress suivant, à la suite duquel ils sont devenus extrêmement faibles, ce qui peut être mis en rapport avec laspect dépérissant des arbres.

Cette plus mauvaise réaction à la sécheresse de 1959-1964 dans les placettes d est à mettre en relation avec une plus faible vigueur des arbres au début de ce stress par rapport à ceux des placettes $\mathrm{s}$. Cette moindre vigueur semble la conséquence de moins bonnes conditions d'alimentation en eau, en raison généralement d'un nombre plus faible d'éclaircies relativement récentes, parfois de conditions topographiques plus défavorables.

On suppose donc que le dépérissement du sapin dans les Vosges a été déclenché par ces deux épisodes rapprochés de sécheresse climatique, dans des stations où les conditions hydriques étaient défavorables. Peut-être cxistait-il, en plus, des facteurs prédisposants, dont la pollution atmosphérique.
\end{abstract}

Mots clés: Sapin pectiné, Vosges, dépérissement, accroissements annuels, sécheresse, éclaircies.

\section{Introduction}

Le dépérissement des forêts, qui est devenu une préoccupation mondiale, a été constaté en France en premier lieu dans les Vosges, sur le sapin (Abies alba Mill.), en 1982. Si d'autres essences et régions ont été touchées depuis lors, le dépérissement du sapin dans les Vosges reste dans notre pays le symbole de cet inquiétant problème. 
Parmi les nombreuses études engagées dans le cadre du programme DEFORPA pour tenter d'expliquer le dépérissement des forêts, beaucoup concernent les Vosges. Aucune n'a encore permis de mettre en évidence de façon certaine le ou les facteurs en cause. Une hypothèse assez répandue fait intervenir conjointement plusieurs facteurs, en tout premier lieu la pollution atmosphérique et ses conséquences directes sur le feuillage et indirectes par l'intermédiaire d'une acidification des sols, mais aussi les accidents climatiques et les maladies (SCHütt \& Cowling, 1985).

Lorsqu'on désire comprendre les raisons des différences observées dans l'état de santé des divers peuplements de sapin dans les Vosges, on se heurte à une difficulté majeure ; en effet, de nombreux facteurs du milieu varient en même temps : conditions de pollution, climat. altitude, sol. topographie, exposition, sans parler de l'âge des peuplements et de leurs caractéristiques sylvicoles.

La méthode utilisée dans l'étude dont nous rendons compte ci-après est destinée à faciliter l'interprétation des différences d'état de santé entre peuplements ; il s'agit de comparer, par couples, des placettes situées à proximité l'une de l'autre: ainsi le niveau de pollution et les conditions climatiques sont homogènes pour une situation donnée.

\section{Méthodes utilisées; placettes retenues}

\subsection{Les placettes (méthode des "couples")}

Nous avons recherché des groupes de deux placettes, situées géographiquement le plus près possible l'une de l'autre. Chacune est constituée en principe d'un ensemble de 6 arbres. Les deux placettes de chaque couple devaient avoir des aspects très tranchés : être l'une très saine (s), l'autre très nettement dépérissante (d) (les arbres correspondant respectivement à la classe 1 et à la moitié la plus sévèrement endommagée de la classe 3 dans l'échelle DEFORPA prenant en compte les pourcentages de pertes d'aiguilles).

Après une prospection assez longue, nous avons retenu tous les cas correspondant assez bien au but recherché, en l'occurence 8 couples (tableau 1). Pour 6 d'entre eux, les deux placettes sont situées de telle façon que climat et niveau de pollution sont sans doute pratiquement identiques ; pour les 2 autres. il peut exister une certaine différence entre les deux placettes.

Tous les couples sont situés dans la moitié nord des Vosges et sur le versant lorrain.

Les âges moyens dans les placettes ne sont souvent pas très différents ; dans 12 placettes sur les 16 l'âge est de $94 \pm 14$ ans.

Les roches-mères sont généralement identiques dans les deux placettes d'un même couple. Elle comprennent : grès vosgien, grès intermédiaire, permien, conglomérat, granite, rhyolithes, grauwacke, en place et en colluvium. Les sols varient entre sol brun acide et sol podzolique, et sont tous nettement acides. Dans les deux placettes d'un même couple, le sol peut être identique ou différent ; dans ce dernier cas, le plus acide correspond tantôt à la placette dépérissante, tantôt à la placette saine. 


\begin{tabular}{|c|c|c|c|c|c|c|c|c|c|c|c|c|c|c|c|}
\hline & ${ }^{x}$ & 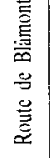 & $\underset{\nabla}{\bar{m}}$ & 文 & $x x$ & 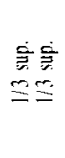 & 产㖓 & 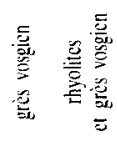 & 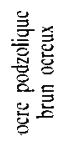 & $\cong \Xi$ & $\ddot{m}$ & $\stackrel{\Rightarrow}{=}$ & $\stackrel{x}{\stackrel{x}{g} \vec{c}}$ & 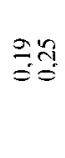 & 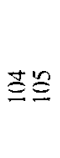 \\
\hline $\begin{array}{l}\dot{s} \\
-\end{array}$ & $\tilde{5}$ & 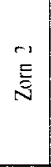 & 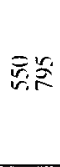 & $\geqslant \omega$ & $\stackrel{c}{N}=$ & 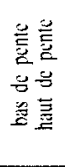 & 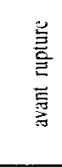 & 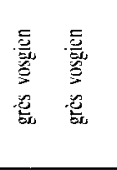 & 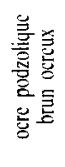 & 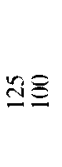 & §き & $\stackrel{0}{\circ}$ & $\stackrel{\infty}{\stackrel{\infty}{\Xi}}$ & $\stackrel{\square=}{9}$ & $\overline{2} \stackrel{3}{3}$ \\
\hline 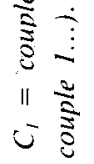 & $E$ & $\overline{\mathrm{E}}$ & $\underset{x}{\bar{x}} \bar{x}$ & $\geqslant \geqslant$ & $\simeq \approx$ & 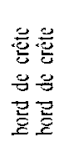 & 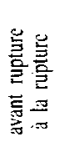 & 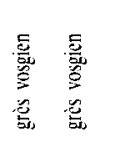 & 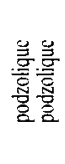 & २尺 & $₹ 8$ & $\stackrel{\square}{0}$ & 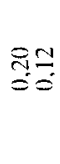 & $\stackrel{+}{ \pm}=$ & 2む \\
\hline 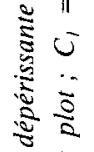 & U & 訔 & 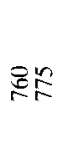 & $2 \xi$ & $n \cong$ & 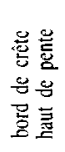 & & 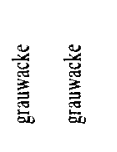 & 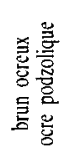 & 象孚 & v্ষে & 32 & ڤ્वे & 궁응 & $\overrightarrow{i n} \tilde{\infty}$ \\
\hline 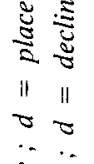 & U & 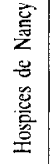 & 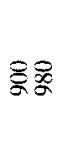 & $z z$ & $\underline{\infty}$ & 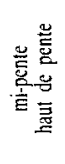 & 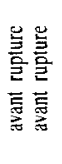 & 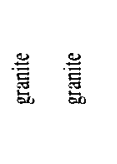 & 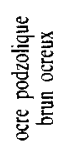 & 88 & 88 & 39 & 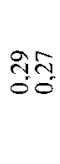 & $\begin{array}{l}09 \\
00\end{array}$ & $2 \infty$ \\
\hline 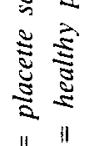 & 0 & 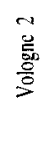 & $\begin{array}{l}\vec{x} \mathscr{x} \\
\text { and }\end{array}$ & $z z$ & $\infty \stackrel{\infty}{.}$ & 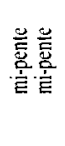 & & 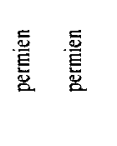 & 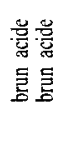 & ฌর & 品年 & $=0$ & 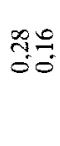 & $\begin{array}{l}08 \\
08 \\
0\end{array}$ & $\infty 8$ \\
\hline 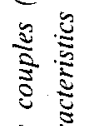 & $\widetilde{U}$ & $\begin{array}{l}\bar{y} \\
\overline{0} \\
\frac{0}{5} \\
\overline{5}\end{array}$ & $\overline{\mathbb{x}} \overline{\mathbb{x}}$ & $n z$ & $\mathrm{NCH}$ & 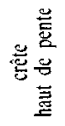 & & 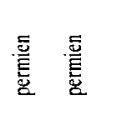 & 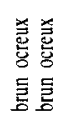 & $\sigma \curvearrowleft$ & Rin & $\vec{c}=$ & ్तㄹ & $\stackrel{\infty}{\stackrel{0}{0}}$ & $\mp \infty$ \\
\hline 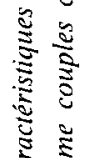 & $\bar{u}$ & $\begin{array}{l}\text { 営 } \\
\text { 离 } \\
\frac{5}{2}\end{array}$ & 胥总 & $\geqslant \geqslant$ & $a 0$ & 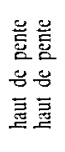 & & 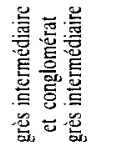 & 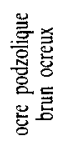 & $\cong$ & वृत्त & $\stackrel{0}{\circ}$ & $\frac{25}{30}$ & $\frac{9}{50}$ & 응 \\
\hline 㫄 & & $\begin{array}{l}\sum \\
0 \\
z\end{array}$ & $\begin{array}{l}\widehat{\Xi} \\
\bar{\Xi} \\
\stackrel{\Xi}{\Xi}\end{array}$ & 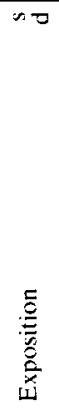 & 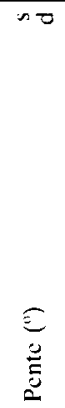 & 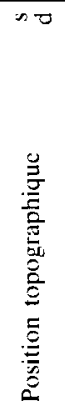 & 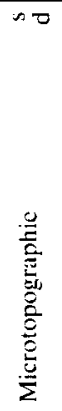 & 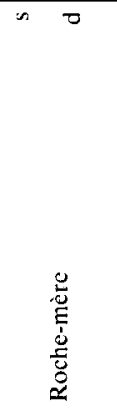 & $\begin{array}{l}\overline{0} \\
0 \\
0 \\
0 \\
0 \\
0 \\
0\end{array}$ & 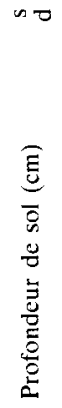 & 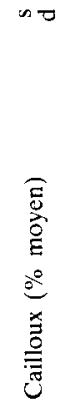 & 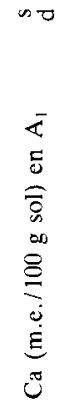 & 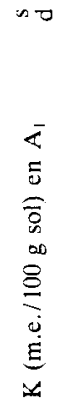 & 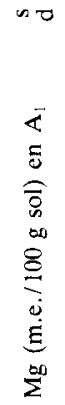 & 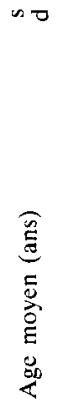 \\
\hline
\end{tabular}




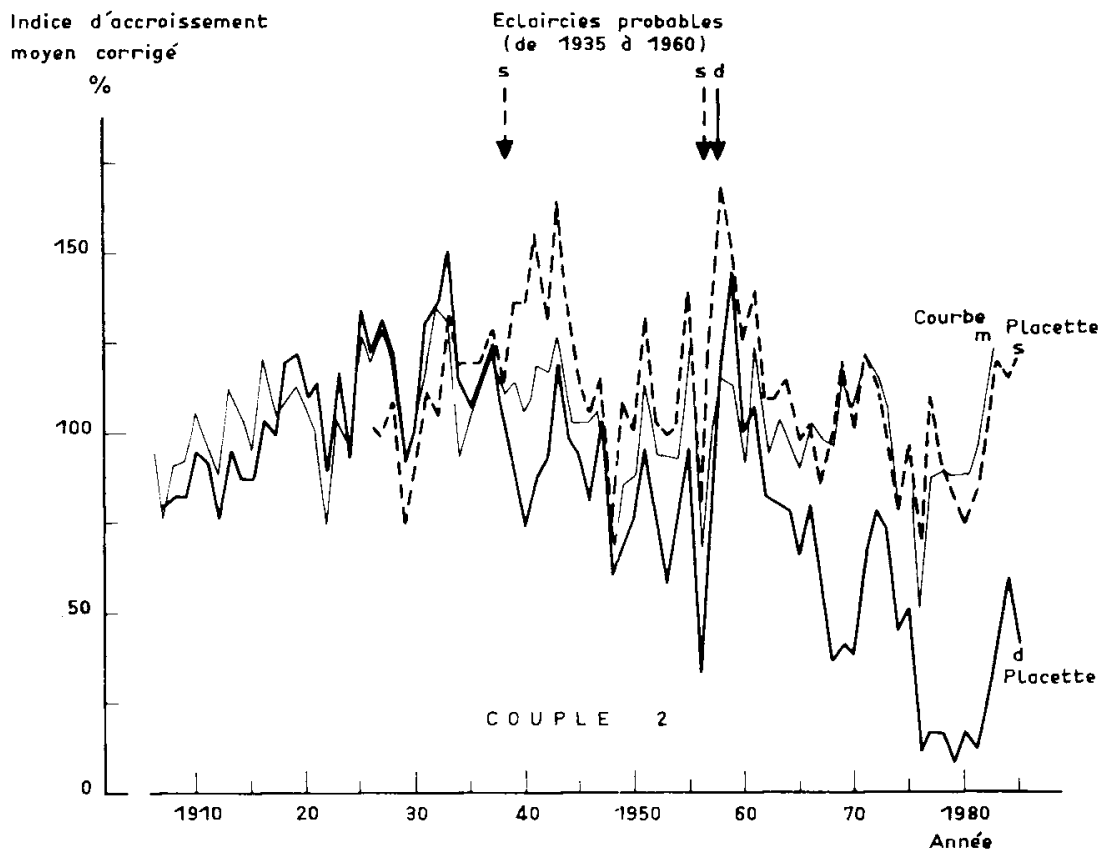

FIi. 1

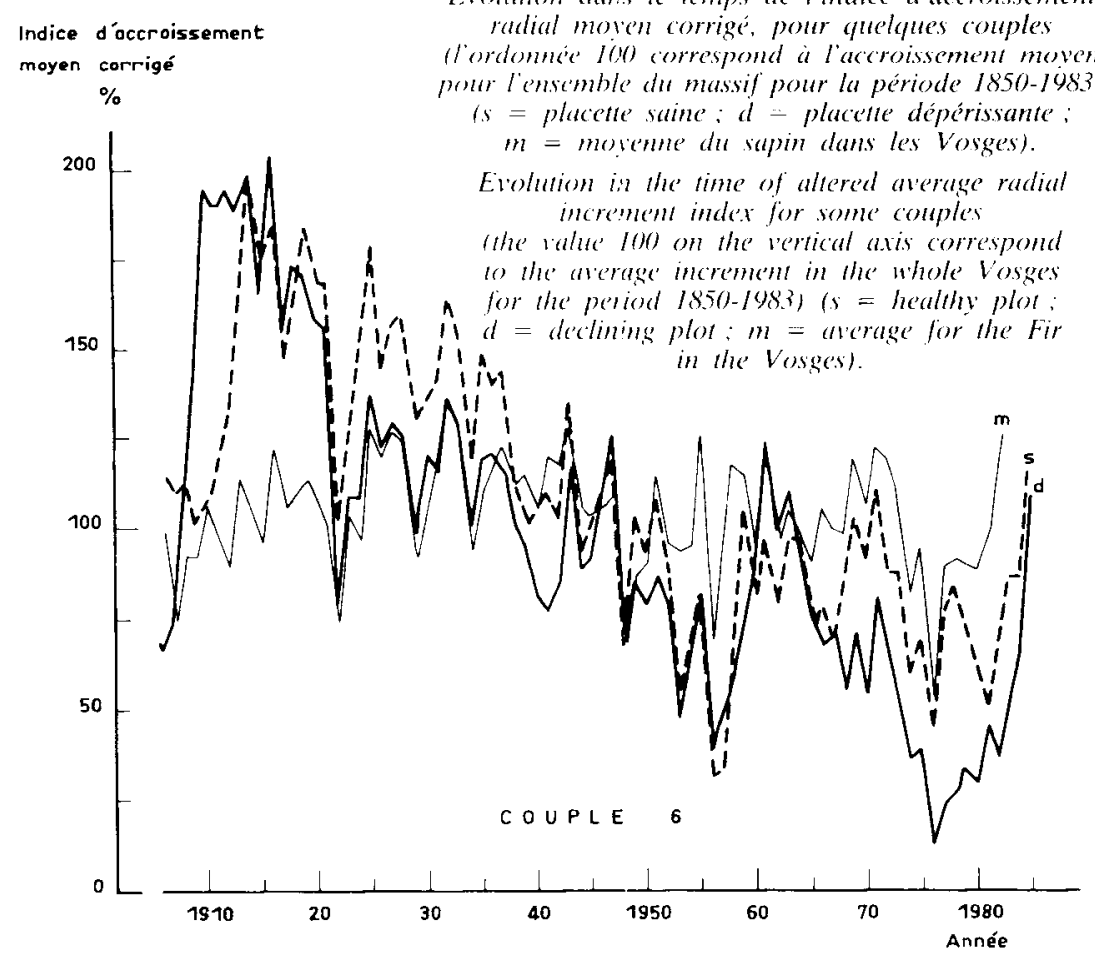



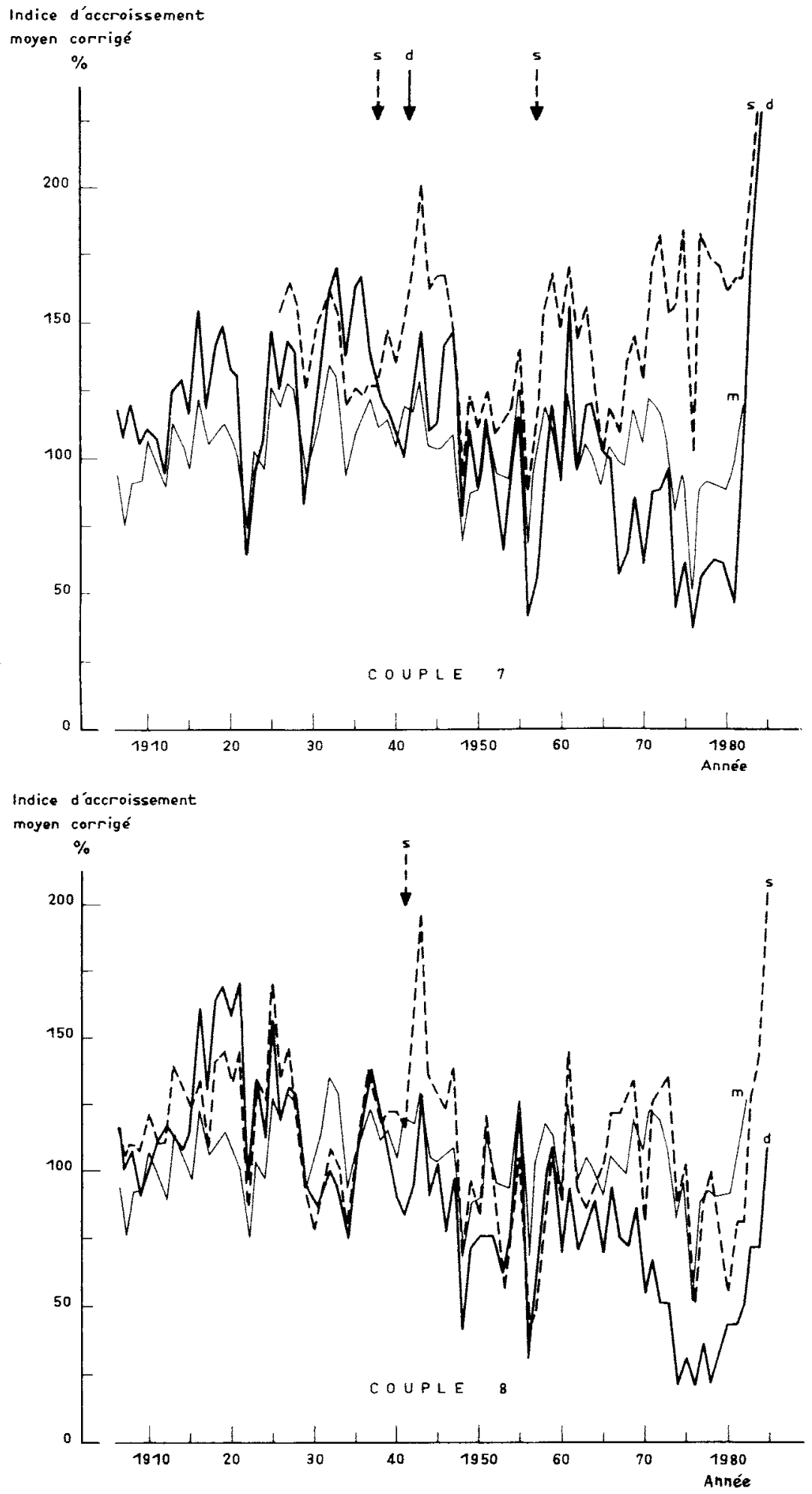


\subsection{L'interprétation}

A partir de carottes de sondage prélevées «à cœur", une courbe de croissance radiale moyenne (pour les 6 arbres) en fonction du temps a été tirée pour chaque placette ; elle représente en fait les croissances annuelles modifiées de façon à éliminer l'effet de l'âge (BEcker, 1987) (voir fig. 1 certaines de ces courbes). La comparaison des croissances permet d'estimer les "vigueurs " relatives dans les différentes placettes, notamment dans les deux d'un même couple. Cette vigueur est un indice objectif de l'état de santé des arbres. On peut par ailleurs logiquement penser qu'aspect dépérissant des arbres et très faible croissance pendant plusieurs années consécutives constituent deux phénomènes dont l'origine, quelle qu'elle soit, est la même ; cela avait été d'ailleurs le cas lors du dépérissement du chêne il y a quelques années (BEcker \& LÉVY, 1982).

Nous disposons également d'une courbe $(m)$ de croissance moyenne du sapin dans les Vosges (corrigée également en fonction de l'âge), établie par l'un de nous (BEcKer, 1987) à partir de 196 placettes choisies aléatoirement, indépendamment du dépérissement.

Les chiffres de croissance (corrigée) que nous citerons seront en fait des indices (obtenus à partir de mesures effectuées sur agrandissement des courbes de croissance). Ces indices peuvent être positifs ou négatifs selon que l'accroissement est supérieur ou inférieur à l'accroissement moyen pour l'ensemble du massif pour la période 1850-1983, représenté sur les figures par l'ordonnée 100.

\section{Résultats}

\subsection{Début de la perte de vigueur dans les placettes dépérissantes: origine et facteur " déclenchant » du dépérissement}

On peut supposer que les variations de croissance annuelle dans les placettes sont surtout induites par deux facteurs essentiels :

- les conditions climatiques annuelles. Le modèle de FriTTs (1966) montre ainsi comment une réduction des précipitations aboutit à une réduction de l'épaisseur des cernes ; cette dernière est fortement corrélée avec les conditions climatiques de l'année en cours et celles de l'année précédente (Zahner \& Donnelly, 1967) ; la figure 2 donne à titre indicatif les totaux pluviométriques au poste météorologique de Strasbourg-Entzheim, situé à proximité (mais à l'extérieur) du massif des Vosges. Une même tendance (pour les croissances annuelles) existe effectivement en général dans l'ensemble du massif; on peut le constater en comparant les courbes des placettes saines (s) ou dépérissantes (d) à la courbe moyenne (m). Mais les conditions climatiques ne sont réellement identiques que pour les deux placettes d'un même couple, ce qui facilite l'interprétation de leurs différences de croissance ;

- les diminutions de concurrence au niveau des différents arbres de la placette, qu'elles soient d'origine accidentelle ou sylvicole. Par commodité, nous les qualifierons toutes « d'éclaircies». 


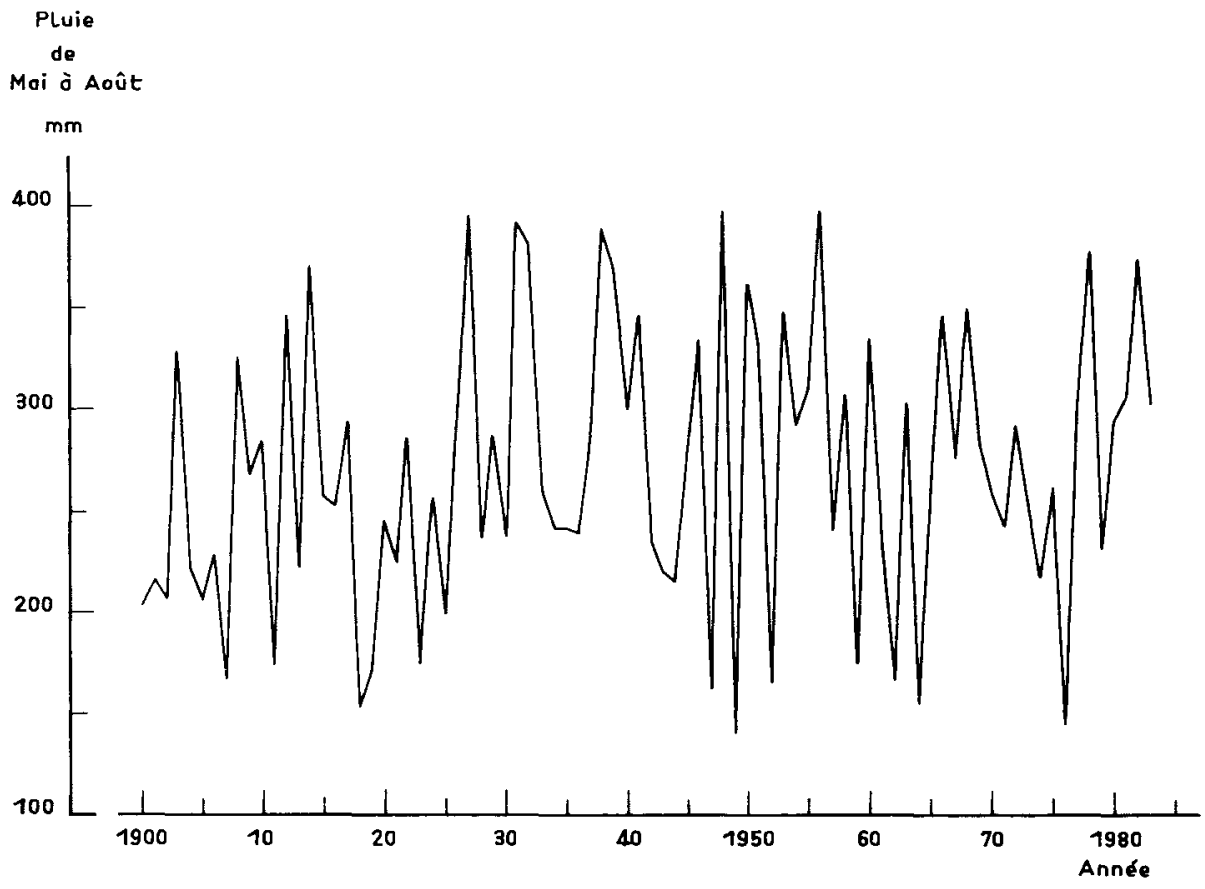

FIG. 2

Totaux pluviométriques de mai à août au poste météorologique de Strasbourg-Entzheim. Rainfall sums from may to august at Strasbourg-Entzheim meteorological station.

\subsection{Années à faible croissance}

Un premier examen des courbes de croissance depuis 30 ans permet de dégager les points suivants :

- toutes les placettes ont un creux de croissance en 1956, mais la croissance repart dès 1957 ;

- de même, en 1974 et surtout en 1976, l'accroissement est partout (relativement) faible ; la reprise de croissance en 1977 est toujours bien plus élevée dans les placettes $s$ que dans les placettes $d$;

- enfin, une période débutant en 1959 correspond à une nette diminution dans les accroissements annuels. Ce phénomène concerne 12 placettes sur les 16 en 1960, 14 placettes en 1962 (dont toutes les d), 13 placettes en 1965 (dont toutes les d). En 1967, les accroissements sont assez faibles dans quelques placettes s, faibles dans toutes les placettes $d$ : une ségrégation apparaît donc entre les deux types de placettes.

Ces différentes années sont-elles caractérisées par des conditions climatiques particulières ?

- 1956 a vu une période exceptionnelle de gelées (fin d'hiver) ;

- 1974 et surtout 1976 sont connues pour leur sécheresse ; 
- 1959-1965 correspond à une période comprenant plusieurs années sèches (19591962-1964) (fig. 2).

\subsection{Différenciation entre les placettes $s$ et $d$; origine $d u$ dépérissement}

Nous venons de voir que les sécheresses rapprochées entre 1959 et 1964 sont peutêtre à l'origine d'une différence de comportement entre les placettes d et s. Il apparaît en fait que depuis 1965 (ou plus tôt, selon le couple), les accroissements annuels dans toutes les placettes $s$ ont toujours été supérieurs à leurs homologues de la placette d correspondante. Les écarts entre les accroissements de s et de d d'un même couple se creusent entre 1966 et 1968, avec presque toujours un paroxysme situé en 1967 ou 1968. Ensuite, ces écarts fluctuent, généralement sans beaucoup se réduire; ils atteignent un nouveau maximum à la suite de la sécheresse de 1976, presque toujours en 1977.

Même si au cours des 25 années précédentes les accroissements annuels dans les placettes s étaient assez souvent plus ou moins supérieurs à ceux des placettes $\mathrm{d}$ (nous en verrons la cause), nous estimons que ce ralentissement relatif de croissance dans les placettes d par raport aux placettes s à la suite des trois années sèches situées entre 1959 et 1964 a constitué la première manifestation générale et très marquée du processus qui a finalement conduit au dépérissernent.

Les futures placettes dépérissantes auraient donc été tellement atteintes par le stress que, contrairement aux placettes s, elles n'ont pas récupéré une fois le stress disparu et ont continué à perdre de leur vigueur. Elles ne s'étaient ainsi pas rétablies lorsque la nouvelle vague de sécheresse est apparue en 1974 et 1976 ; ce deuxième stress, affectant des arbres encore affaiblis, a entraîné pendant quelques années des accroissements extrêmement faibles dans les placettes $d$, ce qui confirme la liaison entre ce dernier phénomène et l'aspect dépérissant des arbres. Les mortalités qui sont intervenues par ailleurs sur le sapin dans les Vosges correspondent très vraisemblablement au même processus général.

Le tableau 2 permet de se faire une idée quantitative (relative) de ces phénomènes (ces chiffres sont donnés à titre d'exemple: d’autres ànées de référence auraient fourni des résultats aussi démonstratifs).

On peut ainsi faire les constatations suivantes:

- en 1968, toutes les placettes s sont plus vigoureuses que les placettes $\mathrm{d}$ correspondantes. L'indice le plus fort parmi les placettes d (-23) est même inférieur à l'indice le plus faible parmi les placettes s ( -13 ) (lignes 1 et 2 ) ;

- à cette même date, toutes les placettes $\mathrm{s}$ avaient mieux réagi que les placettes $\mathrm{d}$ correspondantes, après la fin du stress des années 1959 à 1964, l'année 1963 étant prise comme année de référence : en effet, tous les chiffres sont positifs (ligne 5) ;

- la vigueur moyenne (somme des indices) dans les placettes $\mathrm{d}$ au cours des 10 ans ayant précédé le $2^{\mathrm{c}}$ stress est bien inférieure à celle des placettes $\mathrm{s}$; cela est vrai dans chaque couple. De plus, la vigueur moyenne la plus élevée parmi les placettes d ( -173$)$ est inférieure à la vigueur moyenne la plus faible parmi les placettes s $(-128)$ (lignes 6 et 7 ) ;

- il en est de même si l'on compare les vigueurs moyennes de 1965 à 1979, c'està-dire des 15 ans ayant précédé les premières observations sur le dépérissement (lignes 8 et 9 ). 


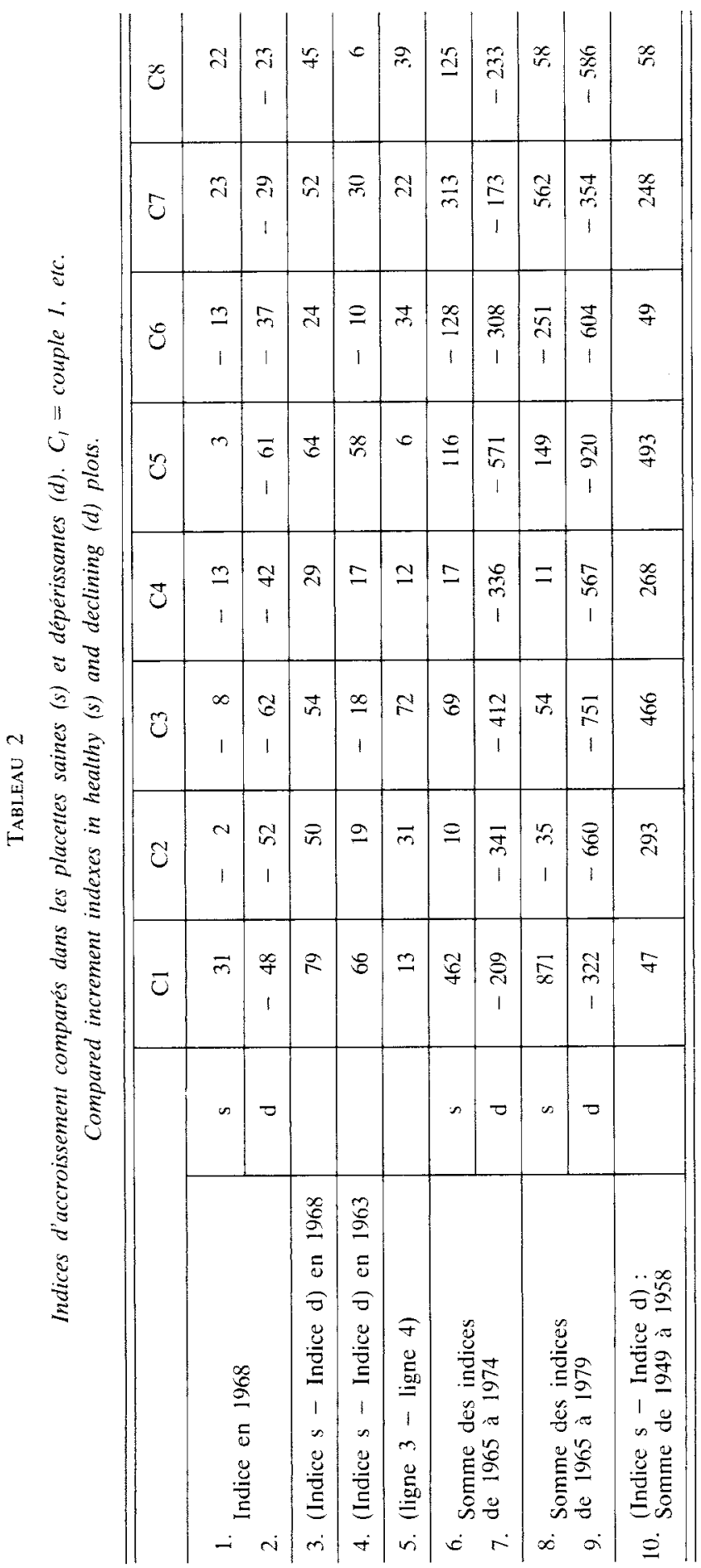


3.13. Est-il anormal, d'un point de vue purement climatique, qu'un "dépérissement" soit apparu à cette époque?

Aucun dépérissement de cette ampleur n’a été signalé en France dans le passé. En ce qui concerne les placettes étudiées, il y a eu parfois des tendances à des " décrochements " dans les accroissements de certaines d'entre elles, mais ce phénomène est généralement beaucoup moins net que dans la période qui nous intéresse; de plus, chaque cas ne concernait qu'un petit nombre de placettes à la fois.

Les conditions climatiques étaient-elles donc plus favorables au cours de la première moitié du $20^{\circ}$ siècle ? Nous avons vu que le dépérissement actuel " s'explique " par la proximité de deux périodes, chacune constituée par une suite (peu interrompue) de 2 ou 3 années sèches (1959-1964 et 1974-1976). Les données météorologiques (poste de Strasbourg-Entzheim, (fig. 2)) témoignent de la présence d'années sèches auparavant (depuis 1880, date depuis laquelle on dispose de données régulières: BECKER, 1987), mais de loin pas aussi rapprochées que celles de la période 1959 à 1976 (une exception, la période 1947-1952, où d'ailleurs des dépérissements importants ont été signalés). On peut ainsi facilement concevoir que les arbres affectés par une sécheresse avaient eu le temps de reprendre vigueur avant l'apparition de la suivante.

Le dépérissement actuel ne paraît donc pas anormal, d'un point de vue purement climatique.

\subsection{Causes de la ségrégation entre peuplements sains et dépérissants}

\subsection{Etat de vigueur lors de l'apparition du stress de 1959 à 1964}

On peut logiquement supposer que les arbres ont dépéri dans les placettes d, et non dans les placettes $s$, parce qu'ils étaient moins vigoureux au moment où le stress des années 1959 à 1964 est intervenu (ou parce que les conditions locales d'alimentation en eau étaient plus défavorables). Si l'on évalue cette vigueur par la croissance radiale cumulée au cours des 10 années précédant ce stress (1949-1958), on constate qu'effectivement, pour chacun des couples, la vigueur au moment du stress est plus élevée dans la placette $s$ que dans la placette $d$ (tableau 2, ligne 10: tous chiffres positifs).

Cette différence de vigueur était cependant, pour chaque couple, sensiblement plus faible que celle qui a fait suite au stress. Au cours de certaines années entre 1949 et 1965, les accroissements dans les deux placetles de chaque couple étaient même pratiquement identiques, ce qui ne s'est plus jamais produit ultérieurement.

\subsection{Origine de la moindre vigueur des arbres avant le stress dans les placettes $d$}

Nous avons vu que les accroissements annuels dépendaient beaucoup de l'alimentation en eau. Les conditions climatiques étant identiques dans les deux placettes de chaque couple, l'origine de différences d'alimentation en eau des arbres, donc sans doute de croissance, dans ces deux placettes ne peut se trouver que parmi les facteurs suivants : concurrence, réserve utile maximum des sols (liée à la profondeur du sol, à la quantité de cailloux, à la texture), topographie tet microtopographie.

Les conditions d'alimentation minérale pourraient également jouer un certain rôle dans les différences de vigueur entre placettes; on ne constate cependant rien de net dans l'ensemble des 8 couples, la placette dépérissante pouvant être selon le cas plus 
riche ou plus pauvre que la placette saine (cf. les types de sol et les teneurs en cations, au tableau 1).

Le problème se pose donc ainsi : un ou plusieurs des 3 facteurs suivants, conditions de concurrence avant 1959, sol, topographie, est-il en défaveur des placettes d par rapport à leur homologue s?

\subsection{Conditions de concurrence avant 1959}

La comparaison d'une courbe de croissance avec la courbe moyenne $\mathbf{m}$ pour les Vosges permet de déceler les années où le peuplement a sans doute subi une éclaircie : il se produit alors une augmentation de croissance relative (par rapport à la courbe $\mathrm{m}$ ) très nette, et qui se poursuit pendant plusieurs années (fig. 1).

On s'aperçoit de cette façon qu'au cours des 25 années ayant précédé le stress de 1959-1964, le bilan du nombre d'éclaircies est bien plus favorable pour les placettes $\mathrm{s}$ que pour les d. En totalité, pour les placettes s, dix éclaircies probables (de plus, dans la placette $\mathrm{s}$ du couple 5, les arbres n'ont apparemment jamais souffert de concurrence) ; pour les placettes d, quatre éclaircies probables. En dehors du cas spécial du couple 5 , le nombre d'éclaircies est toujours plus élevé, pour chaque couple, dans la placette s ( 2 ou 1 éclaircie par placette), que dans la placette d ( 1 ou 0$)$, sauf pour le couple 6 (aucune éclaircie apparente). De plus, la plupart des placettes s ont eu une éclaircie peu avant le stress ou au début de celui-ci, entre 1957 et 1961 , ce qui n'est pas le cas des placettes $d$.

\subsection{Topographie et sol}

Les capacités au champ n'étant probablement pas très différentes dans les deux placettes de chaque couple en raison d'une variation de texture qui reste faible, les estimations des profondeurs de sol (et d'enracinement) et des pourcentages de cailloux, effectuées sur des profils de sol, devraient permettre de comparer les deux placettes du point de vue des réserves maximum des sols en eau utilisable.

L'examen du tableau 1 montre que, pour deux couples, sol ou topographie sont très sensiblement différents dans les deux placettes, et cela en faveur de la placette $s$ : ce sont les couples 4 (pourcentage de cailloux beaucoup plus élevé dans d) et 6 (placette s située avant une rupture de pente, placette d après). Il est possible, mais de façon moins évidente, que pour d'autres couples la réserve en eau utile du sol soit aussi en faveur de la placette $s$ (couples 1, 3, 7). Pour certains couples, les conditions d'alimentation en eau peuvent paraitre en défaveur de la placette s, mais la différence n'est jamais très accentuée.

La topographie est ainsi incontestablement plus favorable dans la placette s du seul couple où cette placette n'avait apparemment pas plus bénéficié d'éclaircies que la placette d. On peut rappeler à cet égard que la position topographique, en liaison avec l'approvisionnement latéral en eau, constitue le facteur du milieu auquel est le plus lié la production d'une espèce exigeante en eau comme le frêne (Devauchelife \& Lévy, 1977 ; LE GofF \& LÉvY, 1984).

On peut donc finalement affirmer que, lors de lapparition du stress des années 1959-1964, la moindre vigueur dans la placette d par rapport à la placette s de chaque couple était liée à des conditions d'alimentation hydrique moins bonnes, en raison d'une plus forte concurrence entre arbres ou/et de conditions topographiques (ou de 
sol) plus défavorables. C'est cette moindre vigueur, ou bien simplement le fait que les conditions locales d'alimentation hydrique étaient moins bonnes, qui explique sans doute que les arbres ont moins résisté au stress dans les placettes $d$ que dans les placettes $\mathrm{s}$; il est impossible de différencier ces deux causes possibles, car elles sont très liées, l'une étant la conséquence de l'autre.

\section{Conclusion}

La méthode des «couples » et l'étude dendrochronologique ont permis de rendre très vraisemblable l'explication suivante :

- le processus du dépérissement dans les placettes "dépérissantes » (d) de chaque couple fait suite à deux périodes assez rapprochées caractérisées chacune par deux ou trois années sèches très voisines : 1959-1964 et 1974-1976. Les arbres, contrairement à ceux des placettes "saines" (s), n'avaient pas récupéré leur vigueur lorsque le deuxième stress est apparu ;

- cette plus forte réaction au $1^{\text {er }}$ stress (1959-1964) dans les placettes d est liée à une plus faible vigueur des arbres, que l'on peut facilement mettre en relation avec une concurrence plus importante entre individus (dans la plupart des cas) (à laquelle s'ajoute parfois un sol à réserve moins élevée en eau utile) ou avec des caractéristiques microtopographiques plus désavantageuses ; en somme, avec des conditions d'alimentation hydrique plus défavorables.

Si l'on tente de généraliser ces résultats, et bien qu'il ne s'agisse que « d'observations ", donc qu'aucune liaison de causalité ne puisse être affirmée, on peut logiquement supposer que le dépérissement du sapin dans les Vosges a été déclenché par ces épisodes rapprochés de sécheresse climatique, dans des stations où les conditions d'alimentation hydrique étaient défavorables.

En dehors de ce facteur « déclenchant ", peut-être existait-il aussi des facteurs «prédisposants », notamment la pollution à longue distance, par son action sur le feuillage ou par acidification des sols, mais aucune certitude n'existe à l'heure actuelle sur son rôle précis dans le phénomène de dépérissement observé en Europe occidentale.

Dans un passé récent ou plus ancien, plusieurs auteurs ont mis en avant la sécheresse comme facteur très important dans les phénomènes de dépérissement. Ainsi pour HePting (1963) le dépérissement sur plusieurs essences aux U.S.A. dans les années 1930 fut consécutif à des sécheresses prolongées. LARSEN (1981) a constaté, dans des essais de provenances de sapin au Danemark, des dépérissements consécutifs aux sécheresses de 1975 et 1976, mais dont étaient indemnes les sujets provenant des régions les plus sèches (S et SE de l'aire naturelle) ; les conclusions de Clauser (1981) sont très semblables. Enfin, Cramer \& Cramer-Middendorf (1984) remarquent que les périodes de crises passées, dans différentes régions et sur diverses essences, notamment le sapin en Forêt Noire, correspondent à des séries de cycles végétatifs secs. Tous ces auteurs restent cependant fortement minoritaires parmi l'ensemble de ceux qui ont étudié le dépérissement des forêts, et ont été très peu suivis dans leurs conclusions jusqu'à présent. 
L'explication que nous proposons mériterait d'être testée (par exemple par utilisation de la même méthode) sur d'autres espèces et dans d'autres régions et pays. Cela ne concerne pas les dépérissements qui interviennent notamment dans certains pays d'Europe de l'Est à proximité de zones fortement industrialisées, et dont l'origine est évidemment la pollution à courte distance.

Signalons enfin, en complément de l'étude générale sur l'ensemble du massif (BECKER, 1987), que l'examen des courbes de croissance dans nos placettes confirme de manière indéniable que la phase de dépérissement du sapin dans les Vosges est terminée. Les arbres paraissent se rétablir dans presque toutes nos placettes dépérissantes; on y constate en effet une reprise de croissance tout à fait spectaculaire, généralement depuis 1983.

Sur un plan pratique, il se dégage de nos résultats l'utilité d'une réflexion approfondie sur la sylviculture du sapin dans les Vosges. Si l'on veut à l'avenir mieux mettre les peuplements à l'abri de crises comparables à celle que nous venons de connaître, il apparaît souhaitable de maintenir un état de concurrence raisonnable et, si possible, adapté aux conditions locales d'approvisionnement en eau.

\section{Summary \\ Silver fir decline in the Vosges: essential part of water nutrition shortages}

The current forest decline has been noted in France first on the Silver Fir in the Vosges. It is often thought now that several factors are responsible : air pollution (acting on leaves or by soil acidification), climate, diseases...

We have used a method which makes easier the interpretation of health conditions differences between stands. We have thus chosen 8 couples of plots, each plot consisting of 6 trees. In each couple, one plot (s) is very healthy, the other (d) very declining (important loss of leaves) ; the two plots are generally closed to each other; thus, the level of air pollution and climate conditions are about the same in both plots.

The plots characteristics appear on table 1. The soils range from acid brown soil to podzolic soil. The more acid and poorer soil in each couple correspond sometimes to the (s) plot, sometimes to the (d) plot.

Average annual radial growth curves, altered to get rid of the age effect, have been drawn ; on these curves, annual increments are in the form of an index (fig. 1). We also have the Fir average growth curve in the Vosges (fig. 1).

We can see on these curves, during the last 25 years, two periods with low increments in almost all plots ; they correspond each to 2 or 3 « dry » years : 1959, 1962 and $1964 ; 1974$ and mainly 1976 (fig. 2). But annual increments in all (d) plots went on to be low for a long time after 1965 : the trees in those plots were undergoing the after-effects on the 1959 to 1964 drought (table 2); unlike in the (s) plots, their increments were still low when the 1974 to 1976 drought occured; then the increments became even lower in the (d) plots for a few years, which can be related to the trees declining appearance.

In relation with the worse reaction to the 1959-1964 droughts in the (d) plots, we note that in each couple the trees in that plot were less sturdy than those in the (s) plot when the drought started (the strength is assessed from the radial growth sum for the previous 10 or 15 years).

That lower strength in the (d) plots comes probably from worse water supply conditions. Indeed, it seems that almost all the (s) plots have undergone more thinnings ( 2 or 1$)$ during the 25 years before the 1959-1964 droughts than the (d) plot in the same couple (1 or 0$)$; therefore, competition for water was probably lower in the (s) plots; in one couple, it is the lateral water 
supply in the (s) plot that is more lavourable than in the (d) plot, because of the topographical conditions.

It may be therefore assessed that the Silver Fir decline in the Vosges was set off by those two near periods of climatic drought (1959-1964 and 1974-1976) in sites where water supply conditions were bad. Perhaps there were in addition predisposing factors, in particular long range air pollution, but there is no certainty about its real part in the declining.

We can see on the (d) plots curves a dramatic growth resumption, generally from 1983.

Key words : Silver Fir, Vosges, decline. annual increments, drought, thinnings.

\section{Références bibliographiques}

Becker M., 1987. Bilan de santé actuel et rétrospectif du sapin (Abies alba Mill.) dans les Vosges. Etude écologique et dendrochronologique. Am. Sci. For. 44 (4).

Beckle M., Lévy G., 1982. Le dépéristement du chêne en forế de Tronçais. Les causes écologiques. Ann. Sci. For., 39 (4), 439-44t.

Cinusir F., 1981. Ancora sulla moria dellabete hianco. Monti e Boschi, 32 (6), 95-97.

Devauchelle R., Lévy G., 1977. Propriétés stationnelles et croissance du frêne dans l'Est de la France. Ann. Sci. For., 34 (3), 231-244.

Cramer H.H., Cramer-Middenidorf M.. 1984. Studies on the relationships between periods of damage and factors of climate in the Middle European forests since 1851. PflanzenschuzNachrichten Bayer, 37 (2), $208-334$.

Frirts H.C., 1966. Growth rings of trees: their correlation with climate. Sciences, 154, p. 973.

Herting G.H., 1963. Climate and forest discases. Annu. Rev. Phylopathol. 1, $31-50$.

LARSEN J. Bo.. 1981. Waldbauliche und ertragskundliche Erfahrungen mit verschiedenen Provenienzen der Weisstanne (Abies alba Mill.) in Däncmark. Forstwiss. Centralblatt, $100(3 / 4)$, 275-286.

Lf. Gorf N., Lévy G., 1984. Productivité du frêne en région Nord-Picardic. B. Etude des relations entre la productivité et les conditions de milicu. Am. Sci. For., 41 (2), 135-170.

Schütt P., Cowling E.B., 1985. Waldsterben, a general decline of forests in Central Europe : symptoms, development and possible causes. Plant disease, 69 (7), 548-558.

ZAHNER R., DONNELY J.R.. 1967. Refining correlations of rainfall and radial growth in young red pinc. Ecology, 48, p. 525. 